\title{
Towards the Development of Cell Permeable Macrocyclic Scaffolds: Probing the Structural Requirements for Enhancing Cellular Uptake
}

\author{
Simon Vézina-Dawod ${ }^{1,3}$, Sophie Fortin ${ }^{1,3}$, Marie Perrin ${ }^{1,3}$, \\ Louis-Jean Bordeleau ${ }^{2,3}$, Stéphane Gobeil ${ }^{2,3}$, and Eric Biron ${ }^{1,3}$ \\ ${ }^{1}$ Faculty of Pharmacy, Université Laval, Quebec (QC), G1V 0A6, Canada; ${ }^{2}$ Department of Molecular Medicine, \\ Université Laval, Quebec (QC), GIV OA6, Canada ${ }^{3} \mathrm{CHU}$ de Québec Research Centre, CHUL, \\ Quebec (QC), G1V 4G2, Canada
}

\section{Introduction}

Peptide macrocycles are useful tools in chemical biology and for the development of therapeutic compounds. Compared to their linear counterparts, cyclic peptides are more resistant to proteases and their increased conformational rigidity makes them tighter-binding to a given macromolecule $[1,2]$. However, the relatively high polarity of the secondary amide bonds through the cyclic peptide backbone often results in poor cellular uptake. Since many therapeutic targets are found inside cells, drugs often need to cross multiple hydrophobic membranes to reach their site of action. To cross a membrane, a molecule must first break its interactions with water to go into the hydrophobic media. In many bioactive cyclic peptides, some amide bonds undergo intramolecular hydrogen bonding (IHB), thus internalizing the polarity $[3,4]$. Other amide bonds are free to interact with water and bring an energetic cost to cross the membrane in a passive way. $N$-Methylation of free amide bonds (not involved in IHB) is a promising approach to improve cellular uptake [5,6]. Another approach is to use peptoid residues ( $N$-substituted glycines) to replace and mimic peptide bonds. Compared to peptides, the side chains migration to the backbone nitrogens increases proteolytic resistance and decreases the global polarity $[7,8]$. However, the replacement of the chiral $\mathrm{C} \alpha$ to $\mathrm{CH}_{2}$ and the display of tertiary amide bonds lead to a lost in conformational definition as $N$-alkylglycine oligomers are more flexible. Considerable efforts have been achieved to rigidify peptoids, by incorporating rigid monomers that induce dihedral angle preferences. Amongst these monomers, $N$-arylglycine was reported to significantly rigidify linear and cyclic peptoids [9]. Kirshenbaum and coworkers have shown that electronic repulsion is occurring between the amide bond oxygen and the aromatic ring, inducing a strong energetic preference for the trans-amide bond [9]. With the aim of developing macrocyclic scaffolds that could mimic the functional and conformational space found in peptide structures, we were interested in studying the relationships between the incorporation of rigid $N$-aryl monomers into cyclic peptoids, the induced conformations and their effects on cellular uptake.

\section{Results and Discussion}

For the study, the cyclic peptide motif $\mathrm{c}\left[\mathrm{Xaa}^{5}-\mathrm{Phe}^{6}-\mathrm{Pro}^{1}-\mathrm{Phe}^{2}{ }_{-} \mathrm{Xaa}^{3}-\mathrm{Xaa}^{4}\right]$ was selected for its ability to induce a type II' $\beta$-turn upon amino acids 3 and 4 . Based on this motif, the sequence $c\left[\mathrm{Glu}^{5}-\mathrm{Phe}^{6}\right.$ Pro $^{1}-\mathrm{Phe}^{2}-\mathrm{DTyr}^{3}-\mathrm{Lys}^{4}$ (Dns)] was chosen as model peptide 1. Next, different modifications such as $\mathrm{N}$-methylation or the introduction of peptoid residues were performed on the model peptide $\mathbf{1}$ to evaluate and compare their impact on cellular uptake (Table 1).

Table 1. Sequences of the synthesized peptide and peptoid analogs for the cellular uptake study.

\begin{tabular}{|c|c|c|}
\hline & Cyclic N-methylated analogs & Cyclic peptoid analogs \\
\hline$\overline{2}$ & $\mathrm{c}[(N-\mathrm{Me})$ Glu-Phe-Pro-Phe-DTyr-Lys(Dns)] & 7 c[Nlys(Dns)-Ntyr-Nphe-Pro- $N$ phe- $N$ glu $]$ \\
\hline 3 & c[Glu-(N-Me)Phe-Pro-Phe-DTyr-Lys(Dns)] & 8 c [Nglu-Nphe-Pro-Nphe-Ntyr-Nlys(Dns) $]$ \\
\hline 4 & c[Glu-Phe-Pro-(N-Me)Phe-DTyr-Lys(Dns)] & $9 \mathrm{c}[N$ glu-Nphg-Pro-Nphe-Ntyr-Nlys(Dns) $]$ \\
\hline 5 & c[Glu-Phe-Pro-Phe-(N-Me)DTyr-Lys(Dns)] & 10 c $[N$ glu-Nphe-Pro-Nphg-Ntyr-Nlys(Dns) $]$ \\
\hline 6 & c[Glu-Phe-Pro-Phe-DTyr-(N-Me)Lys(Dns)] & 11 c[Nglu-Nphg-Pro-Nphg-Ntyr-Nlys(Dns)] \\
\hline
\end{tabular}


A first series of $N$-methylated analogs was prepared by standard solid phase peptide synthesis on 2'-chlorotrityl polystyrene resin using the procedure described by Biron, et al. for selective $\mathrm{N}$-methylation on solid support and HATU as coupling reagent [10]. After cleavage from the resin with a HFIP solution, the $N$-methylated peptides were successively cyclized with PyBOP, deprotected with a solution of TFA and labeled on the lysine side chain with a dansyl moiety in solution to afford analogs 2-6. To prepare peptoid analogs 7-11, a dansyl labeled Nlys monomer was synthesized in solution and coupled to the resin during oligomerization on solid-support. Briefly, $o$-NBS-Nlys(Dns)$\mathrm{OH}$ was synthesized from commercial glycine tert-butyl ester by protection of the amine with $o$-nitrobenzenesulfonyl group (oNBS) followed by $N$-alkylation with $N$-(4-bromobutyl)phtalimide . After removal of the phtalyl protecting group, the side chain amine was labeled with dansyl chloride followed by tert-butyl ester cleavage with TFA to afford the $N^{\varepsilon}$-labeled $N^{\alpha}$-oNBS- $N^{\alpha}$-substituted glycine monomer. After coupling the monomer to the solid support, peptoid analogs 7-11 were prepared by the submonomer approach (Figure 1) [11]. In the case of $N$ phg residues, due to electronic deactivation of the aniline submonomer, bromide displacement was performed over $16 \mathrm{~h}$ instead of $30 \mathrm{~min}$ to ensure complete reaction and bromoacetylation on $N$-arylglycine $90 \mathrm{~min}$ instead of $30 \mathrm{~min}$. Coupling of Fmoc-Pro-OH on $\mathrm{Nphg}$ residues was achieved using bis(trichloromethyl)carbonate (BTC) to generate the acyl chloride in situ. Among the various coupling reagents like HATU, DIC and others that have been tested, BTC was significantly the most efficient one. After cleavage from resin with a HFIP solution, head-to-tail cyclisation and side chain deprotection were performed in solution as described above to afford cyclic peptoid analogs 7-11. To evaluate the effect of cyclisation on cellular uptake, a linear peptoid 12 and a linear analog of the model peptide 13 were also synthesized.

After their purification and characterization by LC-MS, the ability of compounds 1-13 to penetrate inside cells was evaluated by flow cytometry. The cellular uptake study was performed by incubating human cervical carcinoma (HeLa) cells with compounds 1-13 at a final concentration of $50 \mu \mathrm{M}$ or DMSO alone and TAT peptide (Dns-YGRKKRRQRRR) as negative and positive controls, respectively. After incubation for $2 \mathrm{~h}$ at $37^{\circ} \mathrm{C}$, the cells were washed, trypsinized, fixed, and resuspended. The level of cellular uptake was evaluated in flow cytometry using median fluorescence and the standard deviation from triplicates gave the error (Figure 2). The results were compared to model peptide 1 and showed that $N$-alkylation has a major impact on cellular uptake. As expected, $N$-methylated analogs 2-6 yielded variable uptake results, depending on the $N$-methyl group position.
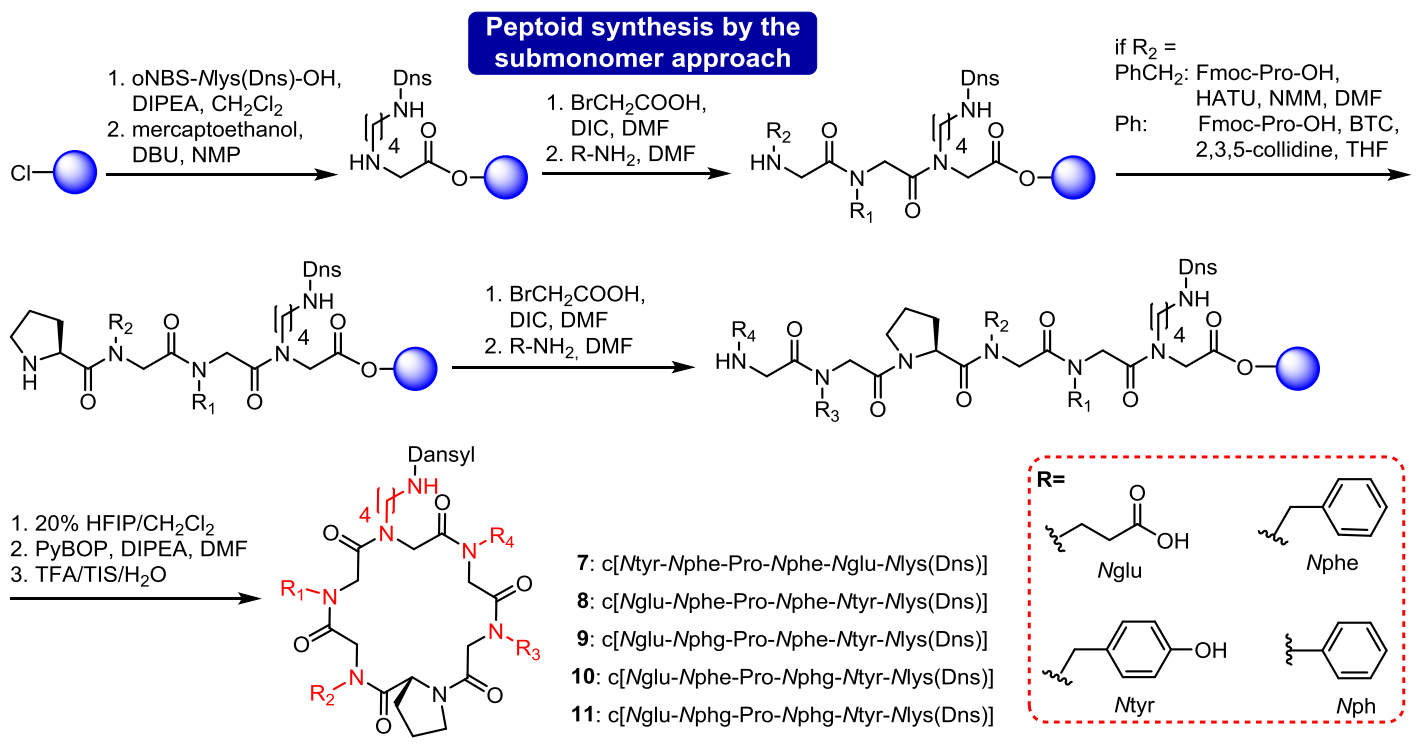

Fig. 1. Synthesis of the cyclic peptoid analogs 7-11. 
Compared to model peptide 1 , a loss of $41 \%$ in cellular uptake was observed for the $N$-methylated Glu analog 2 while no significant difference was observed for $N$-methylation on $\mathrm{Phe}^{6}(3)$. On the other hand, analogs with $N$-methylated $\mathrm{Phe}^{2}(\mathbf{4}), \mathrm{Tyr}(5)$ and Lys (6) residue showed an increase in cellular uptake of 196, 255 and $251 \%$, respectively. These results suggest that some positions are more sensible to $N$-alkylation than others and that their modification lead to a loss of IHB or an important conformational change. It is reasonable to think that $\mathrm{N}-\mathrm{H}$ bond in position 5 might undergo IHB and amide $\mathrm{N}-\mathrm{H}$ in position 2, 3 and 4 are more susceptible to interact with water. No significant difference was observed between the full peptoid $\mathbf{8}$ and the retropeptoid 7, but compared to compound 1, they presented increased uptake of $235 \%$ and $264 \%$, respectively.

Interestingly, compared to cyclic peptoid $\mathbf{8}$, substitution of $N$ phe $^{2}$ by a $N$-phenylglycine $(N \mathrm{phg})$ resulted in a gain of $162 \%$ for compound $\mathbf{1 0}$ while the same substitution at position 6 yielded a loss of $66 \%$ for compound 9. Substitution of $N$ phe residues at positions 2 and 6 by $N$ phg (11) gave intermediate result with a small loss of $16 \%$ compared to cyclic peptoid $\mathbf{8}$. When compared to model peptide 1, the cyclic peptoid $\mathbf{1 0}$ showed a $383 \%$ increase in cellular uptake. By comparison, with a $N$ phg at position 6 , the cellular uptake for the cyclic peptoid analog 9 was comparable to model peptide 1. These results suggest that rigidification of the peptoid scaffold with an $N$-aryl residue can significantly increase the cellular uptake and that its position in the macrocycle is very important. Finally, linear analogs $\mathbf{1 2}$ and $\mathbf{1 3}$ are slightly above the negative control and the results showed that cyclisation of peptide 13 enhanced the cellular uptake by $203 \%$ (1 vs 13) while cyclisation of peptoid 12 increased uptake by $487 \%$ (8 vs 12). No significant difference was observed between linear peptide and peptoid oligomers, suggesting that cyclisation and overall conformation have a greater impact on cellular uptake than only $N$-substitution. From this study, showing nearly a 5 fold increase in cellular uptake compared to its peptide counterpart, the most promising candidate for cell penetration is the $N$-aryl analog $\mathbf{1 0 .}$

In summary, a series of macrocyclic $N$-alkylated peptide and peptoid analogs labeled with a dansyl moiety was synthesized and their ability to penetrate cells evaluated by flow cytometry. The results showed that the amide bond at position 2 in the model peptide 1 seems to play a very important role in the cell penetration capability. Indeed, its modification by $N$-methylation, $N$-substitution and $N$-arylation yielded significant increase in cellular uptake. $N$-Methylation of the $\mathrm{Phe}^{2}$, Tyr or Lys residue yielded 2-2.5 fold increases in cellular uptake.

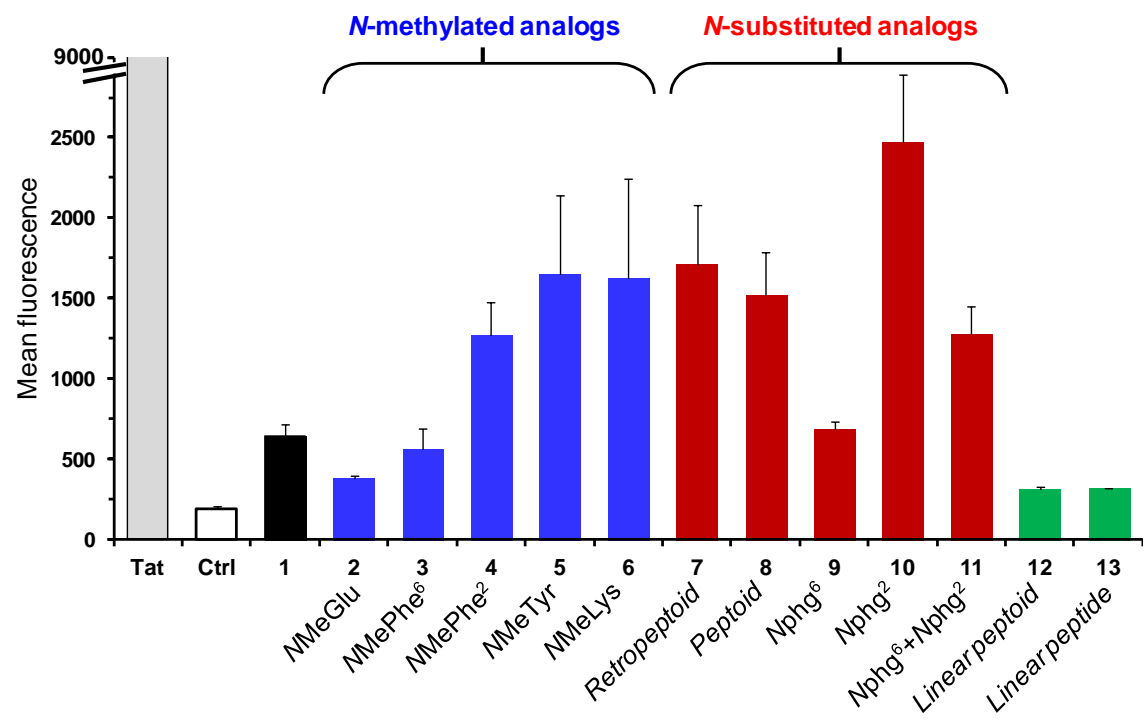

Fig. 2. Comparative cellular uptake of compounds 1-13 on HeLa cells as measured by flow cytometry with excitation at $360 \mathrm{~nm}$. Emission was monitored with 525/50 band-pass filter and fluorescence intensity is expressed as median fluorescence. 
The cyclic peptoid analogs showed also a 2.5 fold increase in cellular uptake but the most significant improvement was observed when the peptoid macrocycle was rigidified by the introduction of an $\mathrm{N}$-aryl residue. Here again the position of the modification is important and the best result was obtained when the $\mathrm{N}$-aryl residue was incorporated at position 2 . In this case a 5 fold increase in cellular uptake was observed. These results strongly suggest that the conformation of a peptide-based macrocyclic compound plays a critical role in its ability to penetrate cells in a passive manner. The relation between conformation and the ability to cross membranes has also been reported for $N$-methylated peptides $[5,6,12]$. The position of the modification has an impact on the macrocycle's conformation that will affect IHB and the total polarity surface area. The described study showed that the ability of a cyclic peptide to penetrate cells can be rapidly and significantly improved by doing the appropriate modifications. Compared to its peptide counterpart, a 5 fold increase in cellular uptake has been observed for the $N$-arylated cyclic peptoid 10. Finally, conformational studies are currently underway to characterize the structural changes in the analogs and define a relation between the modifications and their impact on the overall conformation.

\section{Acknowledgments}

Simon Vézina-Dawod and Sophie Fortin thank the Fond d'enseignement et de recherche de la Faculté de pharmacie de l'Université Laval (FER) and the National Sciences and Engineering Research Council of Canada (NSERC) for research scholarships. This work was supported by the NSERC of Canada (371503-2010).

\section{References}

1. Adessi, C., Soto, C. Curr. Med. Chem. 9, 963-978 (2002), http://dx.doi.org/10.2174/0929867024606731

2. Tyndall, J.D., Nall, T., Fairlie, D.P. Chem. Rev. 105, 973-999 (2005), http://dx.doi.org/10.1021/cr040669e

3. Alex, A., Millan, D.S., Perez, M., Wakenhut, F., Whitlock, G.A. MedChemComm 2, 669 (2011), http://dx.doi.org/10.1039/c1md00093d

4. Kuhn, B., Mohr, P., Stahl, M. J. Med. Chem. 53, 2601-2611 (2010), http://dx.doi.org/10.1021/jm100087s

5. Biron, E., et al. Angew. Chem. Int. Ed. 47, 2595-2599 (2008), http://dx.doi.org/10.1002/anie.200705797

6. White, T.R., et al. Nat. Chem. Biol. 7, 810-817 (2011), http://dx.doi.org/10.1038/nchembio.664

7. Miller, S.M., Simon, R.J., Ng, S., Zuckermann, R.N., Kerr, J.M., Moos, W.H. Bioorg. Med. Chem. Lett. 4, 2657-2662 (1994), http://dx.doi.org/10.1016/S0960-894X(01)80691-0

8. Kwon, Y.U., Kodadek, T. Chem. Biol. 14, 671-677 (2007), http://dx.doi.org/10.1016/j.chembiol.2007.05.006

9. Shah, N.H., Butterfoss, G.L., Nguyen, K., Yoo, B., Bonneau, R., Rabenstein, D.L., Kirshenbaum, K. J. Am.

Chem. Soc. 130, 16622-16632 (2008), http://dx.doi.org/10.1021/ja804580n

10. Biron E, Chatterjee J, Kessler H. J. Peptide Sci. 12, 213-219 (2006), http://dx.doi.org/10.1002/psc.711

11. Zuckerman, R.N., Kerr, J.M., Kent, S.B.H., Moos, W.H. J. Am. Chem. Soc. 114, 10646-10647 (1992), http://dx.doi.org/10.1021/ja00052a076

12. Rezai, T., Yu, B., Millhauser, G.L., Jacobson M.P., Lokey, R.S. J. Am. Chem. Soc. 128, 2510-2511 (2006), http://dx.doi.org/10.1021/ja0563455 